\title{
Georgios Stylogiannis*
}

\section{Hausdorff operators on Bergman spaces of the upper half plane}

https://doi.org/10.1515/conop-2020-0005

Received November 2, 2019; accepted February 6, 2020

Abstract: In this paper we study Hausdorff operators on the Bergman spaces $A^{p}(\mathbb{U})$ of the upper half plane.

Keywords: Hausdorff operator, Bergman space, holomorphic function

MSC: 47B38, 30H20, 46E15

\section{Introduction}

Given a $\sigma$-finite positive Borel measure $\mu$ on $(0, \infty)$, the associated Hausdorff operator $\mathcal{H}_{\mu}$ for suitable functions $f$, is given by

$$
\mathcal{H}_{\mu}(f)(z):=\int_{0}^{\infty} \frac{1}{t} f\left(\frac{z}{t}\right) d \mu(t), \quad z \in \mathbb{U},
$$

where $\mathbb{U}=\{z \in \mathbb{C}: \operatorname{Im} z>0\}$ is the upper half plane. Its formal adjoint, the quasi-Hausdorff operator $\mathcal{H}_{\mu}^{\star}$, in the case of real Hardy spaces $H^{p}(\mathbb{R})$ is

$$
\mathcal{H}_{\mu}^{\star}(f)(z):=\int_{0}^{\infty} f(t z) d \mu(t) .
$$

Moreover, for appropriate functions $f$ and measures $\mu$, they satisfy the fundamental identity:

$$
\widehat{\mathcal{H}_{\mu}(f)}(x)=\int_{0}^{\infty} \widehat{f}(t x) d \mu(t)=\mathcal{H}_{\mu}^{\star}(\widehat{f})(x), \quad x \in \mathbb{R},
$$

where $\widehat{f}$ denotes the Fourier transform of $f$.

The theory of Hausdorff summability started with the paper of Hausdorff [13] in 1921. A little earlier Hurwitz and Silverman in a pioneering paper [14] had clarified the notions of consistency and equivalence of summability methods. The theory then developed in various directions, among them Hausdorff summability of Fourier series and Hausdorff means of Fourier transforms. Much later, Hausdorff summability of power series of analytic functions was considered in [26] and [27] on composition operators and the Cesáro means in Hardy $H^{p}$ spaces. General Hausdorff matrices were considered in [8] and [9]. In [9], the authors studied Hausdorff matrices on a large class of analytic function spaces such as Hardy spaces, Bergman spaces, BMOA, Bloch etc. They characterized which Hausdorff matrices induce bounded operators on these spaces.

Results on Hausdorff operators on spaces of analytic functions were extended in the Fourier transform setting on the real line. Initially in [15], a particular case of a Hausdorff operator was treated, and later, in [12] and [11], the Hausdorff mean of a Fourier-Stieltjes transform was studied on $L^{1}(\mathbb{R})$. The Hausdorff operator

${ }^{\star}$ Corresponding Author: Georgios Stylogiannis: Department of Mathematics, Aristotle University of Thessaloniki, Greece, E-mail: stylog@math.auth.gr 
$H_{\varphi}$, generated by a function $\varphi$ in $L^{1}(\mathbb{R})$, acting on the real Hardy spaces, was initially studied in [24] and [18]. There are many classical operators in analysis which are special cases of the Hausdorff operator for suitable measures $\mu$, such as the classical Hardy operator, its adjoint operator, the Cesáro type operators and the Riemann-Liouville fractional integral operator. See the survey articles [5] and [22] and the references there in. In recent years, there is an increasing interest in the study of boundedness of the Hausdorff operator on the real Hardy spaces and Lebesque spaces (see for example,[1], [2], [3], [6], [16], [17], [19], [20], [21], [23] and [25]).

Motivated by the papers of Hung et al. [16] and [17] we describe the measures $\mu$ that will induce bounded operators on the Bergman spaces $A^{p}(\mathbb{U})$ of the upper half-plane. Next Theorem summarizes the main results (see Theorems 3.5 and 3.7 ):

Theorem 1.1. Let $1 \leq p<\infty$ and $\mu$ be an $\sigma$-finite positive measure on $(0, \infty)$. The Hausdorff operator $\mathcal{H}_{\mu}$ is bounded on $A^{p}(\mathbb{U})$ if and only if

$$
\int_{0}^{\infty} \frac{1}{t^{1-\frac{2}{p}}} d \mu(t)<\infty
$$

Moreover

$$
\left\|\mathcal{H}_{\mu}\right\|_{A^{p}(\mathbb{U}) \rightarrow A^{p}(\mathbb{U})}=\int_{0}^{\infty} \frac{1}{t^{1-\frac{2}{p}}} d \mu(t) .
$$

\section{Preliminaries}

To define single-valued functions, the principal value of the argument is chosen to be in the interval $(-\pi, \pi]$. For $1 \leq p<\infty$, we denote by $L^{p}(d A)$ the Banach space of all measurable functions on $\mathbb{U}$ such that

$$
\|f\|_{L^{p}\left(d A_{a}\right)}:=\left(\frac{1}{\pi} \int_{\mathbb{U}}|f|^{p} d A\right)^{1 / p}<\infty,
$$

where $d A$ is the area measure. The Bergman space $A^{p}(\mathbb{U})$ consists of all holomorphic functions $f$ on $\mathbb{U}$ that belong to $L^{p}(d A)$. Sub-harmonicity yields a constant $C>0$ such that

$$
|f(z)|^{p} \leq \frac{C}{(\operatorname{Im}(z))^{2}}\|f\|_{A^{p}(\mathbb{U})}^{p}, \quad z \in \mathbb{U},
$$

for $f \in A^{p}(\mathbb{U})$ and

$$
\lim _{z \rightarrow \partial \widehat{\mathbb{U}}}(\operatorname{Im}(z))^{2}|f(z)|^{p}=0
$$

for functions $A^{p}(\mathbb{U})$, where $\widehat{\mathbb{U}}:=\overline{\mathbb{U}} \cup\{\infty\}$ (see [7]). In particular, this shows that each point evaluation is a continuous linear functional on $A^{p}(\mathbb{U})$.

The duality properties of Bergman spaces are well known in literature (see [4] and [28]). It is proved that for $1<p<\infty, \frac{1}{p}+\frac{1}{q}=1$, the dual space of the Bergman space $A^{p}(\mathbb{U})$ is $\left(A^{p}(\mathbb{U})\right)^{\star} \sim A^{q}(\mathbb{U})$ under the duality pairing,

$$
\langle f, g\rangle=\frac{1}{\pi} \int_{\mathbb{U}} f(z) \overline{g(z)} d A(z) .
$$

\section{Main Results}

In what follows, unless otherwise stated, $\mu$ is a positive $\sigma$-finite measure on $(0, \infty)$. We start by giving a condition under which $\mathcal{H}_{\mu}$ is well defined. 
Lemma 3.1. Let $1 \leq p<\infty$ and $f \in A^{p}(\mathbb{U})$. If $\int_{0}^{\infty} \frac{1}{t^{1-\frac{2}{p}}} d \mu(t)<\infty$, then

$$
\mathcal{H}_{\mu}(f)(z)=\int_{0}^{\infty} \frac{1}{t} f\left(\frac{z}{t}\right) d \mu(t)
$$

is a well defined holomorphic function on $\mathbb{U}$.

Proof. For $f \in A^{p}(\mathbb{U})$, using (4), we have:

$$
\begin{aligned}
\left|\mathcal{H}_{\mu}(f)(z)\right| & \leq \int_{0}^{\infty} \frac{1}{t}\left|f\left(\frac{z}{t}\right)\right| d \mu(t) \\
& \leq C \frac{\|f\|_{A^{p}(\mathbb{U})}}{\operatorname{Im}(z)^{\frac{2}{p}}} \int_{0}^{\infty} \frac{1}{t^{1-\frac{2}{p}}} d \mu(t)<\infty .
\end{aligned}
$$

Thus $\mathcal{H}_{\mu} f$ is well defined and is given by an absolutely convergent integral, so it is holomorphic.

Lemma 3.2. Let $\lambda>0$ and $\delta>0$. If $g_{\lambda, \delta}(z)=|z+\delta i|^{-\frac{2+\lambda}{p}}$, then

$$
\left(\frac{1}{2}\right)^{2+\lambda} \cdot \frac{1}{\lambda \delta^{\lambda}} \leq\left\|g_{\lambda, \delta}\right\|_{L^{p}(d A)}^{p} \leq 2^{\frac{2+\lambda}{2}} \cdot \frac{1}{\lambda \delta^{\lambda}}
$$

Proof. Using polar coordinates for the integral over $\mathbb{U}$, we find

$$
\begin{aligned}
\left\|g_{\lambda, \delta}\right\|_{L^{p}(d A)}^{p} & =\frac{1}{\pi} \int_{\mathbb{U}}\left|\frac{1}{z+\delta i}\right|^{2+\lambda} d A(z) \\
& =\frac{1}{\pi} \int_{0}^{\pi} \int_{0}^{\infty}\left(\frac{1}{r^{2}+\delta^{2}+2 r \delta \sin (\theta)}\right)^{\frac{2+\lambda}{2}} r d r d \theta .
\end{aligned}
$$

Denote by $I$ the last double integral. Then

$$
\begin{aligned}
I & \leq \int_{0}^{\infty}\left(\frac{1}{r^{2}+\delta^{2}}\right)^{\frac{2+\lambda}{2}} r d r \\
& \leq 2^{\frac{2+\lambda}{2}} \int_{0}^{\infty}\left(\frac{1}{r+\delta}\right)^{2+\lambda}(r+\delta) d r \\
& =2^{\frac{2+\lambda}{2}} \frac{1}{\lambda \delta^{\lambda}} .
\end{aligned}
$$

On the other hand,

$$
\begin{aligned}
I & \geq \int_{0}^{\infty}\left(\frac{1}{r+\delta}\right)^{2+\lambda} r d r \\
& \geq \int_{\delta}^{\infty}\left(\frac{1}{r+\delta}\right)^{2+\lambda} r d r \\
& \geq\left(\frac{1}{2}\right)^{2+\lambda} \int_{\delta}^{\infty}\left(\frac{1}{r}\right)^{2+\lambda} r d r \\
& =\left(\frac{1}{2}\right)^{2+\lambda} \frac{1}{\lambda \delta^{\lambda}},
\end{aligned}
$$

and the assertion follows. 


\subsection{Test functions}

We now consider the test functions which are defined as follows. Let $z=x+i y \in \mathbb{U}$ and

$$
\varphi_{\varepsilon}(z)=\frac{\overline{z+\varepsilon i}}{|z+\varepsilon i|}=\frac{x-i(y+\varepsilon)}{\sqrt{x^{2}+(y+\varepsilon)^{2}}}
$$

and

$$
f_{\varepsilon}(z)=\frac{1}{(z+\varepsilon i)^{\frac{2}{p}+\varepsilon}},
$$

with $\varepsilon>0$ small enough. Note that $\left|f_{\varepsilon}\right| \equiv g_{p \varepsilon, \varepsilon}$ with respect to the notation of Lemma 3.2, and that $\varphi_{\varepsilon}(z)$ lies on the unit circle with $-\pi<\arg \left(\varphi_{\varepsilon}(z)\right)<0$, and the following identity holds

$$
f_{\varepsilon}(z)=\varphi_{\varepsilon}(z)^{\frac{2}{p}+\varepsilon}\left|f_{\varepsilon}(z)\right| .
$$

Let $a, b \in(-\pi, \pi]$ and set $A_{[a, b]}=\{z \in \mathbb{U}: a \leq \arg (z) \leq b\}$, with obvious modifications in the case of $A_{(a, b)}, A_{(a, b]}$ and $A_{[a, b)}$.

Lemma 3.3. The following holds:

(i) If $2<p<\infty$ and $\frac{2}{p}+\varepsilon \leq 1$, then

$$
\left|\operatorname{Re} f_{\varepsilon}(z)\right| \geq\left|\operatorname{Re} \varphi_{\varepsilon}(z)\right|\left|f_{\varepsilon}(z)\right|
$$

for every $z \in A_{\left(0, \frac{\pi}{2}\right]}$.

(ii) If $1<p \leq 2$ and $1<\frac{2}{p}+\varepsilon<2$, then

$$
\left|\operatorname{Im} f_{\varepsilon}(z)\right|>C(p)\left|\operatorname{Im} \varphi_{\varepsilon}(z)\right|\left|f_{\varepsilon}(z)\right|
$$

for every $z \in A_{\left[\frac{\pi}{4}, \frac{\pi}{2}\right]}$.

(ii) If $p=1,0<\theta_{0}<\frac{\pi}{16}$ and $(2+\varepsilon)\left(\frac{\pi}{2}+\theta_{0}\right)<\frac{5 \pi}{4}$, then

$$
\left|\operatorname{Re} f_{\varepsilon}(z)\right|>\left|\operatorname{Re} \varphi_{\varepsilon}(z)\right|\left|f_{\varepsilon}(z)\right|
$$

for every $z \in A_{\left[\frac{\pi}{2}, \frac{\pi}{2}+\theta_{0}\right]}$

Proof. Taking real and imaginary parts we have

$$
\operatorname{Re} f_{\varepsilon}(z)=\left|f_{\varepsilon}(z)\right| \operatorname{Re} \varphi_{\varepsilon}(z)^{\frac{2}{p}+\varepsilon}=\left|f_{\varepsilon}(z)\right| \cos \left(\left(\frac{2}{p}+\varepsilon\right) \theta\right)
$$

and

$$
\operatorname{Im} f_{\varepsilon}(z)=\left|f_{\varepsilon}(z)\right| \operatorname{Im} \varphi_{\varepsilon}(z)^{\frac{2}{p}+\varepsilon}=\left|f_{\varepsilon}(z)\right| \sin \left(\left(\frac{2}{p}+\varepsilon\right) \theta\right),
$$

where $\theta:=\theta(z, \varepsilon)=\arg \varphi_{\varepsilon}(z)$.

(i): It is easy to see that

$$
\begin{aligned}
\left|\operatorname{Re} f_{\varepsilon}(z)\right| & =\left|f_{\varepsilon}(z)\right| \cos \left(\left(\frac{2}{p}+\varepsilon\right) \theta\right) \\
& \geq\left|f_{\varepsilon}(z)\right| \cos (\theta)=\left|\operatorname{Re} \varphi_{\varepsilon}(z)\right|\left|f_{\varepsilon}(z)\right| .
\end{aligned}
$$

(ii): Let $a=a(p)>0$ such that $1<\frac{2}{p}+\varepsilon<a<2$. Since $z \in A_{\left[\frac{\pi}{4}, \frac{\pi}{2}\right]}$ simple geometric arguments imply that $\theta \in\left[-\frac{\pi}{2},-\frac{\pi}{4}\right)$. Moreover $-\pi<-\frac{a \pi}{2}<\left(\frac{2}{p}+\varepsilon\right) \theta<-\frac{\pi}{4}$. This implies that

$$
\min \left\{\sin \left(\frac{a \pi}{2}\right), \frac{\sqrt{2}}{2}\right\}<\left|\frac{\sin \left(\left(\frac{2}{p}+\varepsilon\right) \theta\right)}{\sin (\theta)}\right|<\sqrt{2}
$$


for every $z \in A_{\left[\frac{\pi}{4}, \frac{\pi}{2}\right]}$. We calculate

$$
\begin{aligned}
& \left|\operatorname{Im} f_{\varepsilon}(z)\right|=\left|f_{\varepsilon}(z)\right|\left|\sin \left(\left(\frac{2}{p}+\varepsilon\right) \theta\right)\right|>\min \left\{\sin \left(\frac{a \pi}{2}\right), \frac{\sqrt{2}}{2}\right\}\left|f_{\varepsilon}(z)\right||\sin (\theta)| \\
& =\min \left\{\sin \left(\frac{a \pi}{2}\right), \frac{\sqrt{2}}{2}\right\}\left|\operatorname{Im} \varphi_{\varepsilon}(z)\right|\left|f_{\varepsilon}(z)\right| .
\end{aligned}
$$

This proves (ii) with $C(p)=\min \left\{\sin \left(\frac{a(p) \pi}{2}\right), \frac{\sqrt{2}}{2}\right\}$.

(iii): Since $z \in A_{\left[\frac{\pi}{2}, \frac{\pi}{2}+\theta_{0}\right]}$, we have that $\theta \in\left(-\frac{\pi}{2}-\theta_{0},-\frac{\pi}{2}\right]$. Thus

$$
-\frac{5 \pi}{4}<-\left(\frac{\pi}{2}+\theta_{0}\right)(2+\varepsilon)<(2+\varepsilon) \theta \leq-\frac{\pi}{2}(2+\varepsilon)<-\pi .
$$

This implies that $|\cos ((2+\varepsilon) \theta)|>|\cos (\theta)|$ and therefore

$$
\left|\operatorname{Re} f_{\varepsilon}(z)\right|>\left|\operatorname{Re} \varphi_{\varepsilon}(z)\right|\left|f_{\varepsilon}(z)\right| .
$$

\subsection{Growth estimates}

Let $a, b \in(-\pi, \pi]$ and set

$$
S_{[a, b]}=\{z \in \mathbb{U}: a \leq \arg (z) \leq b,|z| \geq 1\},
$$

be a truncated sector with obvious modifications in the case of $S_{(a, b)}, S_{(a, b]}$ and $S_{[a, b)}$. Since $\mu$ is positive

$$
\operatorname{Re} \mathcal{H}_{\mu}\left(f_{\varepsilon}\right)=\mathcal{H}_{\mu}\left(\operatorname{Re} f_{\varepsilon}\right) \text { and } \operatorname{Im} \mathcal{H}_{\mu}\left(f_{\varepsilon}\right)=\mathcal{H}_{\mu}\left(\operatorname{Im} f_{\varepsilon}\right) .
$$

Note that if $\operatorname{Re} f_{\varepsilon}$ or $\operatorname{Im} f_{\varepsilon}$ have constant sign on some sector $A$, then

$$
\left|\mathcal{H}_{\mu}\left(\operatorname{Re} f_{\varepsilon}\right)(z)\right|=\mathcal{H}_{\mu}\left(\left|\operatorname{Re} f_{\varepsilon}\right|\right)(z) \text { and }\left|\mathcal{H}_{\mu}\left(\operatorname{Im} f_{\varepsilon}\right)(z)\right|=\mathcal{H}_{\mu}\left(\left|\operatorname{Im} f_{\varepsilon}\right|\right)(z)
$$

for every $z \in A$.

Lemma 3.4. Let $1 \leq p<\infty$ and suppose that $\mathcal{H}_{\mu}$ is bounded on $A^{p}(\mathbb{U})$. Then there are $\varepsilon(p)$ and $k(p)$ positive constants such that

$$
\left\|\mathcal{H}_{\mu}\left(f_{\varepsilon}\right)\right\|_{A^{p}(\mathbb{U})}^{p} \geq k(p)\left(\int_{0}^{\frac{1}{\varepsilon}} \frac{1}{t^{1-\frac{2}{p}-\varepsilon}} d \mu(t)\right)^{p} \frac{1}{p \varepsilon},
$$

for every $\varepsilon$ in $(0, \varepsilon(p)]$.

Proof. We will consider three cases for the range of $p$. Note that if $z$ is in a truncated sector $S$, then $z / t$ belongs to the corresponding sector $A$ for every $t>0$.

Case I. Let $2<p<\infty$ and $\varepsilon(p)$ such that $\frac{2}{p}+\varepsilon(p)<1$. Then for every $\varepsilon$ in $(0, \varepsilon(p)]$

$$
\begin{aligned}
\left\|\mathcal{H}_{\mu}\left(f_{\varepsilon}\right)\right\|_{A^{p}(\mathbb{U})}^{p} & \geq\left\|\operatorname{Re} \mathcal{H}_{\mu}\left(f_{\varepsilon}\right)\right\|_{A^{p}(\mathbb{U})}^{p}=\left\|\mathcal{H}_{\mu}\left(\operatorname{Re} f_{\varepsilon}\right)\right\|_{A^{p}(\mathbb{U})}^{p} \\
& \geq \frac{1}{\pi} \int_{S_{\left(0, \frac{\pi}{2}\right]}}\left|\int_{0}^{\infty} \frac{1}{t} \operatorname{Re} f_{\varepsilon}(z / t) d \mu(t)\right|^{p} d A(z) \\
& =\frac{1}{\pi} \int_{S_{\left(0, \frac{\pi}{2}\right]}}\left(\int_{0}^{\infty} \frac{1}{t}\left|\operatorname{Re} f_{\varepsilon}(z / t)\right| d \mu(t)\right)^{p} d A(z) .
\end{aligned}
$$


Denote by $I$ the last integral on $S_{\left(0, \frac{\pi}{2}\right]}$. By (i) of Lemma 3.3 we have

$$
\begin{aligned}
I & \geq \frac{1}{\pi} \int_{S_{\left(0, \frac{\pi}{2}\right]}}\left(\int_{0}^{\frac{1}{\varepsilon}} \frac{1}{t}\left|\operatorname{Re} \varphi_{\varepsilon}(z / t)\right|\left|f_{\varepsilon}(z / t)\right| d \mu(t)\right)^{p} d A(z) \\
& =\frac{1}{\pi} \int_{S_{\left(0, \frac{\pi}{2}\right]}}\left(\int_{0}^{\frac{1}{\varepsilon}}\left(\frac{1}{\sqrt{x^{2}+(y+t \varepsilon)^{2}}}\right)^{\frac{2}{p}+\varepsilon+1} \frac{d \mu(t)}{t^{1-\frac{2}{p}-\varepsilon}}\right)^{p} x^{p} d x d y .
\end{aligned}
$$

We will estimate this integral using polar coordinates. Observe that

$$
\begin{aligned}
\sqrt{x^{2}+(y+t \varepsilon)^{2}} & =\sqrt{r^{2}+2 r t \varepsilon \sin (\theta)+t^{2} \varepsilon^{2}} \\
& \leq \sqrt{r^{2}+2 r t \varepsilon+t^{2} \varepsilon^{2}}, \quad \theta \in\left(0, \frac{\pi}{2}\right] .
\end{aligned}
$$

Also, since $0<t \leq 1 / \varepsilon$ we have $t \varepsilon \leq 1 \leq|z|=r$, so

$$
\sqrt{r^{2}+2 r t \varepsilon+t^{2} \varepsilon^{2}} \leq \sqrt{4 r^{2}}=2 r
$$

and we have

$$
\begin{aligned}
& I \geq \int_{1}^{\infty} \int_{0}^{\frac{\pi}{2}}\left(\int_{0}^{\frac{1}{\varepsilon}}\left(\frac{1}{2 r}\right)^{\frac{2}{p}+\varepsilon+1} \frac{d \mu(t)}{t^{1-\frac{2}{p}-\varepsilon}}\right)^{p} r^{p+1}(\cos (\theta))^{p} \frac{d \theta}{\pi} d r \\
& =k(p, \varepsilon)\left(\int_{0}^{\frac{1}{\varepsilon}} \frac{1}{t^{1-\frac{2}{p}-\varepsilon}} d \mu(t)\right)^{p} \int_{1}^{\infty} \frac{1}{r^{1+p \varepsilon}} d r \\
& =k(p, \varepsilon)\left(\int_{0}^{\frac{1}{\varepsilon}} \frac{1}{t^{1-\frac{2}{p}-\varepsilon}} d \mu(t)\right)^{\frac{1}{p \varepsilon}},
\end{aligned}
$$

where $k(p, \varepsilon)=2^{-p(\varepsilon+1)} \int_{0}^{\frac{\pi}{2}}(\cos (\theta))^{p} \frac{d \theta}{4 \pi}$.

Case II. Let $2<p<\infty$ and $\varepsilon(p)$ such that $1<\frac{2}{p}+\varepsilon(p)<2$. Then for every $\varepsilon$ in $(0, \varepsilon(p)]$

$$
\begin{aligned}
\left\|\mathcal{H}_{\mu}\left(f_{\varepsilon}\right)\right\|_{A^{p}(\mathbb{U})}^{p} & \geq \frac{1}{\pi} \int_{S_{\left[\frac{\pi}{4}, \frac{\pi}{2}\right]} \mid}\left|\int_{0}^{\infty} \frac{1}{t} \operatorname{Im} f_{\varepsilon}(z / t) d \mu(t)\right|^{p} d A(z) \\
& =\frac{1}{\pi} \int_{S_{\left[\frac{\pi}{4}, \frac{\pi}{2}\right]}}\left(\int_{0}^{\infty} \frac{1}{t}\left|\operatorname{Im} f_{\varepsilon}(z / t)\right| d \mu(t)\right)^{p} d A(z) .
\end{aligned}
$$


Denote by $I$ the last integral on $S_{\left[\frac{\pi}{4}, \frac{\pi}{2}\right]}$. By (ii) of Lemma 3.3 we have

$$
\begin{aligned}
& I \geq \frac{C(p)}{\pi} \int_{S_{\left[\frac{\pi}{4}, \frac{\pi}{2}\right]}}\left(\int_{0}^{\frac{1}{\varepsilon}} \frac{1}{t}\left|\operatorname{Im} \varphi_{\varepsilon}(z / t)\right|\left|f_{\varepsilon}(z / t)\right| d \mu(t)\right)^{p} d A(z) \\
& \geq \frac{C(p)}{\pi} \int_{S_{\left[\frac{\pi}{4}, \frac{\pi}{2}\right]}}\left(\int_{0}^{\frac{1}{\varepsilon}}\left(\frac{1}{\sqrt{x^{2}+(y+t \varepsilon)^{2}}}\right)^{\frac{2}{p}+\varepsilon+1} \frac{d \mu(t)}{t^{1-\frac{2}{p}-\varepsilon}}\right)^{p} y^{p} d x d y .
\end{aligned}
$$

Using polar coordinates and working as in Case I, we arrive at the desired conclusion with constant $k(p, \varepsilon)=C(p) 2^{-p(\varepsilon+1)} \int_{\frac{\pi}{4}}^{\frac{\pi}{2}}(\sin (\theta))^{p} \frac{d \theta}{4 \pi}$.

Case III. Let $p=1$ and $\theta_{0}$ as in Lemma 3.3. Let $\varepsilon(1)$ such that $(2+\varepsilon(1))\left(\frac{\pi}{2}+\theta_{0}\right)<\frac{5 \pi}{4}$. Then, for every $\varepsilon$ in $(0, \varepsilon(1)]$

$$
\begin{aligned}
\left\|\mathcal{H}_{\mu}\left(f_{\varepsilon}\right)\right\|_{A^{1}(\mathbb{U})} & \geq \frac{1}{\pi} \int_{S_{\left[\frac{\pi}{2}, \frac{\pi}{2}+\theta_{0}\right]} \mid}\left|\int_{0}^{\infty} \frac{1}{t} \operatorname{Re} f_{\varepsilon}(z / t) d \mu(t)\right| d A(z) \\
& =\frac{1}{\pi} \int_{S_{\left[\frac{\pi}{2}, \frac{\pi}{2}+\theta_{0}\right]}} \int_{0}^{\infty} \frac{1}{t}\left|\operatorname{Re} f_{\varepsilon}(z / t)\right| d \mu(t) d A(z) .
\end{aligned}
$$

Denote by $I$ the last integral on $S_{\left[\frac{\pi}{2}, \frac{\pi}{2}+\theta_{0}\right]}$. By (iii) of Lemma 3.3 we have:

$$
\begin{aligned}
I & \geq \frac{1}{\pi} \int_{S_{\left[\frac{\pi}{2}, \frac{\pi}{2}+\theta_{0}\right]}} \int_{0}^{\frac{1}{\varepsilon}} \frac{1}{t}\left|\operatorname{Re} \varphi_{\varepsilon}(z / t)\right|\left|f_{\varepsilon}(z / t)\right| d \mu(t) d A(z) \\
& =\frac{1}{\pi} \int_{S_{\left[\frac{\pi}{2}, \frac{\pi}{2}+\theta_{0}\right]}} \int_{0}^{\frac{1}{\varepsilon}}\left(\frac{1}{\sqrt{x^{2}+(y+t \varepsilon)^{2}}}\right)^{3+\varepsilon} \frac{d \mu(t)}{t^{-1-\varepsilon}}(-x) d x d y .
\end{aligned}
$$

Using polar coordinates and working as is Case I, we arrive at the desired conclusion with constant $k(1, \varepsilon)=$ $-2^{-(\varepsilon+1)} \int_{\frac{\pi}{2}}^{\frac{\pi}{2}+\theta_{0}} \cos (\theta) \frac{d \theta}{4 \pi}$.

Theorem 3.5. Let $1 \leq p<\infty$. The operator $\mathcal{H}_{\mu}$ is bounded on $A^{p}(\mathbb{U})$ if and only if

$$
\int_{0}^{\infty} \frac{1}{t^{1-\frac{2}{p}}} d \mu(t)<\infty .
$$

Proof. Suppose that

$$
\int_{0}^{\infty} \frac{1}{t^{1-\frac{2}{p}}} d \mu(t)<\infty,
$$


then Lemma 3.1 implies that $\mathcal{H}_{\mu}(f)$ is well defined and holomorphic in $\mathbb{U}$. An easy computation involving the Minkowski inequality shows that for all $1 \leq p<\infty$

$$
\begin{aligned}
\left\|\mathcal{H}_{\mu}(f)\right\|_{A^{p}(\mathbb{U})} & =\left(\int_{\mathbb{U}}\left|\int_{0}^{\infty} \frac{1}{t} f\left(\frac{z}{t}\right) d \mu(t)\right|^{p} d A(z)\right)^{1 / p} \\
& \leq\|f\|_{A^{p}(\mathbb{U})} \int_{0}^{\infty} \frac{1}{t^{1-\frac{2}{p}}} d \mu(t)<\infty .
\end{aligned}
$$

Thus $\mathcal{H}_{\mu}$ is bounded on $A^{p}(\mathbb{U})$.

Conversely, suppose that $\mathcal{H}_{\mu}$ is bounded. Let $f_{\varepsilon}(z)=(z+\varepsilon i)^{-\left(\frac{2}{p}+\varepsilon\right)}$ with $\varepsilon>0$ small enough. By Lemma 3.2

$$
\left\|f_{\varepsilon}\right\|_{A^{p}(\mathbb{U})}^{p} \sim \frac{1}{p \varepsilon \varepsilon^{p \varepsilon}} .
$$

Moreover, Lemma 3.4 implies that there is a constant $k=k(p, \varepsilon)>0$ such that

$$
\begin{aligned}
\left\|f_{\varepsilon}\right\|_{A^{p}(\mathbb{U})}^{p}\left\|\mathcal{H}_{\mu}\right\|_{A^{p}(\mathbb{U}) \rightarrow A^{p}(\mathbb{U})} & \geq\left\|\mathcal{H}_{\mu}\left(f_{\varepsilon}\right)\right\|_{A^{p}(\mathbb{U})}^{p} \\
& \geq k\left(\int_{0}^{\frac{1}{\varepsilon}} \frac{1}{t^{1-\frac{2}{p}-\varepsilon}} d \mu(t)\right)^{p} \frac{1}{p \varepsilon} .
\end{aligned}
$$

Thus by letting $\varepsilon \rightarrow 0$, we have in comparison to (5)

$$
\int_{0}^{\infty} \frac{1}{t^{1-\frac{2}{p}}} d \mu(t)<\infty
$$

In order to compute the norm of $\mathcal{H}_{\mu}$, we will firstly compute the norm of the truncated Hausdorff operator $\mathcal{H}_{\mu}^{\delta}$ given by :

$$
\mathcal{H}_{\mu}^{\delta}(f)(z):=\int_{0}^{\infty} \frac{1}{t} f\left(\frac{z}{t}\right) X_{[\delta, 1 / \delta]}(t) d \mu(t)=\int_{\delta}^{\frac{1}{\delta}} \frac{1}{t} f\left(\frac{z}{t}\right) d \mu(t) .
$$

Proposition 3.6. Let $1 \leq p<\infty$ and $0<\delta<1$. If

$$
\int_{0}^{\infty} \frac{1}{t^{1-\frac{2}{p}}} d \mu(t)<\infty
$$

then $\mathcal{H}_{\mu}^{\delta}$ is bounded with

$$
\left\|\mathcal{H}_{\mu}^{\delta}\right\|_{A^{p}(\mathbb{U}) \rightarrow A^{p}(\mathbb{U})}=\int_{\delta}^{\frac{1}{\delta}} \frac{1}{t^{1-\frac{2}{p}}} d \mu(t) .
$$

Proof. As in Theorem 3.5, an application of Minkowski inequality gives

$$
\left\|\mathcal{H}_{\mu}^{\delta}\right\|_{A^{p}(\mathbb{U}) \rightarrow A^{p}(\mathbb{U})} \leq \int_{\delta}^{\frac{1}{\delta}} \frac{1}{t^{1-\frac{2}{p}}} d \mu(t) .
$$


Let $f_{\varepsilon}(z)=(z+i)^{-\frac{2}{p}-\varepsilon}$ with $\varepsilon>0$ small enough. We calculate

$$
\begin{aligned}
\mathcal{H}_{\mu}^{\delta}\left(f_{\varepsilon}\right)(z) & -f_{\varepsilon}(z) \int_{\delta}^{\frac{1}{\delta}} \frac{1}{t^{1-\frac{2}{p}}} d \mu(t) \\
& =\int_{\delta}^{\frac{1}{\delta}} \frac{1}{t^{1-\frac{2}{p}}}\left(\varphi_{\varepsilon, z}(t)-\varphi_{\varepsilon, z}(1)\right) d \mu(t),
\end{aligned}
$$

where

$$
\varphi_{\varepsilon, z}(t)=\frac{t^{\varepsilon}}{(z+t i)^{\frac{2}{p}+\varepsilon}}
$$

For any $t \in[\delta, 1 / \delta]$, calculus gives

$$
\begin{aligned}
\left|\varphi_{\varepsilon, z}(t)-\varphi_{\varepsilon, z}(1)\right| & \leq|t-1| \sup \left\{\left|\varphi_{\varepsilon, z}^{\prime}(s)\right|: s \in[\delta, 1 / \delta]\right\} \\
& \leq \frac{1}{\delta}\left(\frac{\varepsilon \delta^{\varepsilon-1}}{|z+i \delta|^{\frac{2}{p}+\varepsilon}}+\frac{\left(\frac{2}{p}+\varepsilon\right)(1 / \delta)^{\varepsilon}}{|z+i \delta|^{\frac{2}{p}+\varepsilon+1}}\right),
\end{aligned}
$$

so using the notation of Lemma 3.2,

$$
\left|\varphi_{\varepsilon, z}(t)-\varphi_{\varepsilon, z}(1)\right| \leq \varepsilon \delta^{\varepsilon-2} g_{p \varepsilon, \delta}(z)+\left(\frac{2}{p}+\varepsilon\right)(1 / \delta)^{\varepsilon+1} g_{p(\varepsilon+1), \delta}(z) .
$$

Thus, by (7), (8) and an easy application of Minkowski inequality followed by the triangle inequality, we have:

$$
\begin{aligned}
\| \mathcal{H}_{\mu}^{\delta}\left(f_{\varepsilon}\right)(z) & -f_{\varepsilon}(z) \int_{\delta}^{\frac{1}{\delta}} \frac{1}{t^{1-\frac{2}{p}}} d \mu(t) \|_{A^{p}(\mathbb{U})} \\
\leq & (p, \varepsilon) \int_{\delta}^{\frac{1}{\delta}} \frac{1}{t^{1-\frac{2}{p}}} d \mu(t),
\end{aligned}
$$

where

$$
\Phi(p, \varepsilon)=\varepsilon \delta^{\varepsilon-2}\left\|g_{p \varepsilon, \delta}\right\|_{L^{p}(d A)}+\left(\frac{2}{p}+\varepsilon\right)(1 / \delta)^{\varepsilon+1}\left\|g_{p(\varepsilon+1), \delta}\right\|_{L^{p}(d A)} .
$$

This, together with Lemma 3.2 (recall that $\left|f_{\varepsilon}\right|=g_{p \varepsilon, \varepsilon}$ ), yields

$$
\frac{\left\|\mathcal{H}_{\mu}^{\delta}\left(f_{\varepsilon}\right)(z)-f_{\varepsilon}(z) \int_{\delta}^{\frac{1}{\delta}} \frac{1}{t^{1-\frac{2}{p}}} d \mu(t)\right\|_{A^{p}(\mathbb{U})}}{\left\|f_{\varepsilon}\right\|_{A^{p}(\mathbb{U})}} \leq \frac{\Phi(p, \varepsilon)}{\left\|f_{\varepsilon}\right\|_{A^{p}(\mathbb{U})}} \int_{\delta}^{\frac{1}{\delta}} \frac{1}{t^{1-\frac{2}{p}}} d \mu(t)
$$

and the quantity $\Phi(p, \varepsilon) /\left\|f_{\varepsilon}\right\|_{A^{p}(\mathbb{U})}$ tends to 0 as $\varepsilon \rightarrow 0$. This and (6) imply that

$$
\int_{\delta}^{\frac{1}{\delta}} \frac{1}{t^{1-\frac{2}{p}}} d \mu(t)=\left\|\mathcal{H}_{\mu}^{\delta}\right\|_{A^{p}(\mathbb{U}) \rightarrow A^{p}(\mathbb{U})}
$$

Theorem 3.7. Let $1 \leq p<\infty$. If

$$
\int_{0}^{\infty} \frac{1}{t^{1-\frac{2}{p}}} d \mu(t)<\infty
$$

then

$$
\left\|\mathcal{H}_{\mu}\right\|_{A^{p}(\mathbb{U}) \rightarrow A^{p}(\mathbb{U})}=\int_{0}^{\infty} \frac{1}{t^{1-\frac{2}{p}}} d \mu(t)
$$


Proof. By Theorem 3.5, we have that

$$
\left\|\mathcal{H}_{\mu}\right\|_{A^{p}(\mathbb{U}) \rightarrow A^{p}(\mathbb{U})} \leq \int_{0}^{\infty} \frac{1}{t^{1-\frac{2}{p}}} d \mu(t) .
$$

Minkowski inequality implies that

$$
\left\|\mathcal{H}_{\mu}-\mathcal{H}_{\mu}^{\delta}\right\|_{A^{p}(\mathbb{U}) \rightarrow A^{p}(\mathbb{U})} \leq \int_{(0, \delta) \cup\left(\frac{1}{\delta}, \infty\right)} \frac{1}{t^{1-\frac{2}{p}}} d \mu(t) .
$$

By Proposition 3.6

$$
\int_{\delta}^{\frac{1}{\delta}} \frac{1}{t^{1-\frac{2}{p}}} d \mu(t)=\left\|\mathcal{H}_{\mu}^{\delta}\right\|_{A^{p}(\mathbb{U}) \rightarrow A^{p}(\mathbb{U})}
$$

This, combined with (9), allows us to conclude that

$$
\left\|\mathcal{H}_{\mu}\right\|_{A^{p}(\mathbb{U}) \rightarrow A^{p}(\mathbb{U})} \geq \int_{0}^{\infty} \frac{1}{t^{1-\frac{2}{p}}} d \mu(t)-2 \int_{(0, \delta) \cup(1 / \delta, \infty)} \frac{1}{t^{1-\frac{2}{p}}} d \mu(t) \rightarrow \int_{0}^{\infty} \frac{1}{t^{1-\frac{2}{p}}} d \mu(t)
$$

as $\delta \rightarrow 0$. Hence,

$$
\left\|\mathcal{H}_{\mu}\right\|_{A^{p}(\mathbb{U}) \rightarrow A^{p}(\mathbb{U})}=\int_{0}^{\infty} \frac{1}{t^{1-\frac{2}{p}}} d \mu(t)
$$

\subsection{The quasi-Hausdorff operator}

Let $f, g \in A^{2}(\mathbb{U})$ and assume that $\mathcal{H}_{\mu}$ is bounded on $A^{2}(\mathbb{U})$. Thus

$$
\mu(0, \infty)=\int_{0}^{\infty} d \mu(t)<\infty .
$$

We have

$$
\begin{aligned}
\int_{\mathbb{U}} \int_{0}^{\infty} \frac{1}{t}\left|f\left(\frac{z}{t}\right)\right||g(z)| d \mu(t) d A(z) & \leq\left(\int_{\mathbb{U}}\left(\int_{0}^{\infty} \frac{1}{t}\left|f\left(\frac{z}{t}\right)\right| d \mu(t)\right)^{2} d A(z)\right)^{1 / 2}\|g\|_{A^{2}(\mathbb{U})} \\
& \leq(\mu(0, \infty))^{1 / 2}\|f\|_{A^{2}(\mathbb{U})}\|g\|_{A^{2}(\mathbb{U})}<\infty
\end{aligned}
$$

where we applied the Cauchy-Schwarz and Minkowski inequalities. Therefore

$$
\begin{aligned}
\left\langle\mathcal{H}_{\mu}(f), g\right\rangle & =\int_{\mathbb{U}} \mathcal{H}_{\mu}(f)(z) \overline{g(z)} d A(z) \\
& =\frac{1}{\pi} \int_{\mathbb{U}} f(z) \overline{\int_{0}^{\infty} \operatorname{tg}(t z) d \mu(t) d A(z),}
\end{aligned}
$$

where we applied a change of variables and Fubini's Theorem twice. This means that the adjoint $\mathcal{H}_{\mu}^{\star}$ of $\mathcal{H}_{\mu}$ on $A^{2}(\mathbb{U})$ is: 


$$
\mathcal{H}_{\mu}^{\star}(f)(z)=\int_{0}^{\infty} t f(t z) d \mu(t) .
$$

We will consider $\mathcal{H}_{\mu}^{\star}$ on $A^{p}(\mathbb{U})$ and suppose for a moment that it is well defined for functions in $A^{p}(\mathbb{U})$. Let $\lambda(t)=t^{-1}, t>0$, then $\lambda$ maps $(0, \infty)$ onto $(0, \infty)$ and is measurable. Set $f(t z)=f_{z}(t)$ then

$$
\begin{aligned}
\mathcal{H}_{\mu}^{*}(f)(z) & =\int_{0}^{\infty} t f(t z) d \mu(t) \\
& =\int_{0}^{\infty} t f_{z}(t) d \mu(t) \\
& =\int_{0}^{\infty} \frac{1}{\lambda(t)} f_{z}\left(\frac{1}{\lambda(t)}\right) d \mu(t) \\
& =\int_{0}^{\infty} \frac{1}{t} f\left(\frac{z}{t}\right) d v(t), \\
& =\mathcal{H}_{v}(f)(z)
\end{aligned}
$$

where $d \nu=d \lambda_{\star}(\mu)(t)$ and $\lambda_{\star}(\mu)$ is the push-forward measure of $\mu$ with respect to $\lambda$. By a proof similar to the proof of Lemma 3.1, it is easy to show that if

$$
\int_{0}^{\infty} t^{1-\frac{2}{p}} d \mu(t)<\infty
$$

then $\mathcal{H}_{\mu}^{\star}(f)$ is well defined on $\mathbb{U}$. We can now apply the results of the first part of the paper to have:

Theorem 3.8. Let $1 \leq p<\infty$. The quasi-Hausdorff operator $\mathcal{H}_{\mu}^{*}$ is bounded on $A^{p}(\mathbb{U})$ if and only if

$$
\int_{0}^{\infty} t^{1-\frac{2}{p}} d \mu(t)<\infty
$$

Moreover

$$
\left\|\mathcal{H}_{\mu}^{\star}\right\|_{A^{p}(\mathbb{U}) \rightarrow A^{p}(\mathbb{U})}=\int_{0}^{\infty} t^{1-\frac{2}{p}} d \mu(t) .
$$

Acknowledgments: The author is grateful to the anonymous referee for the careful reading of the paper and for his/her suggestions that have further improved the paper.

\section{References}

[1] L. Aizenberg, E. Liflyand and A. Vidras, Hausdorff operators in Hardy spaces on Cartan type domains in $\mathbb{C}^{n}$. Complex analysis and dynamical systems VI. Part 2, 27-46. Contemp. Math., 667, Israel Math. Conf. Proc., Amer. Math. Soc., Providence, RI, 2016.

[2] L. Aizenberg and E. Liflyand, Hardy spaces in Reinhardt domains, and Hausdorff operators. Illinois J. Math. 53 (2009), no. 4, 1033-1049, doi:10.1215/ijm/1290435337

[3] R. Bandaliyev, and P. Górka, Hausdorff operator in Lebesgue spaces. Math. Inequal. Appl. 22 (2019), no. 2, 657-676, doi.org/10.7153/mia-2019-22-45

[4] S. Ballamoole, J. O. Bonyo, T. L. Miller and V. G. Miller, Cesáro-like operators on the Hardy and Bergman spaces of the half plane. Complex Anal. Oper. Theory 10 (2016), no. 1, 187-203, doi.org/10.1007/s11785-015-0481-8 
[5] J. Chen, D. Fan and S. Wang, Hausdorff Operators on Euclidean Spaces, Appl. Math. J. Chinese Univ. (Ser. B) (4) 28 (2014), 548-564, doi.org/10.1007/s11766-013-3228-1

[6] J. Chen and X. Zhu, Boundedness of multidimensional Hausdorff operators on $H^{1}\left(\mathbb{R}^{n}\right)$, J. Math. Anal. Appl. 409 (2014) 428434, doi.org/10.1016/j.jmaa.2013.07.042

[7] B. R. Choe, H. Koo and W. Smith, Difference of composition operators over the half-plane. Trans. Amer. Math. Soc. 369 (2017), no. 5, 3173-3205, doi.org/10.1090/tran/6742

[8] P. Galanopoulos and A. G. Siskakis, Hausdorff matrices and composition operators. Illinois J. Math. 45 (2001), no. 3, 757-773, doi:10.1215/ijm/1258138149

[9] P. Galanopoulos and M. Papadimitrakis, Hausdorff and quasi-Hausdorff matrices on spaces of analytic functions. Canad. J. Math. 58 (2006), no. 3, 548-579, doi.org/10.4153/CJM-2006-023-5

[10] J. B. Garnett, Bounded analytic functions. Revised first edition. Graduate Texts in Mathematics, 236 (Springer, New York, 2007)

[11] C. Georgakis, The Hausdorff mean of a Fourier-Stieltjes transform. Proc. Amer. Math. Soc. 116 (1992), no. 2, 465-471, doi.org/10.1090/S0002-9939-1992-1096210-9

[12] R. R. Goldberg, Convolutions and general transforms on Lp, Duke Math. J. 27 (1960), 251-259, doi:10.1215/S0012-7094-6002722-8

[13] F. Hausdorff, Summationsmethoden und Momentfolgen I. Math. Z. 9 (1921), 74-109, doi.org/10.1007/BF01378337

[14] W. A. Hurwitz and L. L. Silverman, The consistency and equivalence of certain definitions of summability. Trans. Amer. Math. Soc. 18(1917), 1-20, doi.org/10.1090/S0002-9947-1917-1501058-2

[15] G. H. Hardy, Divergent Series (Oxford, at the Clarendon Press, 1949)

[16] H. D. Hung, L. D. Ky, and T. T. Quang, Norm of the Hausdorff operator on the real Hardy space $H^{1}(\mathbb{R})$. Complex Anal. Oper. Theory 12 (2018), no. 1, 235-245, doi.org/10.1007/s11785-017-0651-y

[17] H. D. Hung, L. D. Ky, and T. T. Quang, Hausdorff operators on holomorphic Hardy spaces and applications. Proc. Roy. Soc. Edinburgh Sect. A (to appear); doi:10.1017/prm.2018.74.

[18] Y. Kanjin, The Hausdorff operators on the real Hardy spaces $H_{p}(\mathbb{R})$. Studia Math. 148 (2001), no. 1, 37-45, doi: 10.4064/sm148-1-4

[19] A. Karapetyants, S. Samko and K. Zhu, A Class of Hausdorff-Berezin Operators on the Unit Disc. Complex Anal. Oper. Theory 13 (2019), no. 8, 3853-3870, doi.org/10.1007/s11785-019-00934-x

[20] A. Lerner and E. Liflyand, Multidimensional Hausdorff operators on the real Hardy space, J. Austr. Math. Soc. 83 (2007), 79-86, doi.org/10.1017/S1446788700036399

[21] E. Liflyand and A. Miyachi, Boundedness of multidimensional Hausdorff operators in $H^{p}$ spaces, $0<p<1$. Trans. Amer. Math. Soc. 371 (2019), no. 7, 4793-4814, doi.org/10.1090/tran/7572

[22] E. Liflyand, Hausdorff operators on Hardy spaces. Eurasian Math. J. 4 (2013), no. 4, 101-141, http://www.mathnet.ru/php/ archive.phtml?wshow=paper\&jrnid=emj\&paperid=147\&option_lang=eng

[23] E. Liflyand and A. Miyachi, Boundedness of the Hausdorff operators in $H^{p}$ spaces, $0<p<1$, Boundedness of the Hausdorff operators in $H^{p}$ spaces, $0<p<1$. Studia Math. 194 (2009), no. 3, 279-292, doi: 10.4064/sm194-3-4

[24] E. Liflyand and F. Moricz, The Hausdorff operator is bounded on the real Hardy space $H^{1}(\mathbb{R})$, Proc. Amer. Math. Soc. 128 (2000), no. 5, 1391-1396, doi.org/10.1090/S0002-9939-99-05159-X

[25] A. R. Mirotin, Boundedness of Hausdorff operators on real Hardy spaces $H^{1}$ over locally compact groups. J. Math. Anal. Appl. 473 (2019), no. 1, 519-533, doi.org/10.1016/j.jmaa.2018.12.065

[26] A. G. Siskakis, Composition operators and the Cesáro operator on $H^{p}$. J. London Math. Soc. (2) 36 (1987), no. 1, 153-164, doi.org/10.1112/jlms/s2-36.1.153

[27] A. G. Siskakis, The Cesáro operator is bounded on $H^{1}$. Proc. Amer. Math. Soc. 110 (1990), no. 2, 461-462, doi: $10.2307 / 2048089$

[28] K. H. Zhu, Operator theory in function spaces. Monographs and Textbooks in Pure and Applied Mathematics, 139, (Marcel Dekker, Inc., New York, 1990) 